\title{
Optical coherence tomography: a novel modality for scrotal imaging
}

\author{
Cole Davis, $M D ;^{*}$ Wayne Kuang, $M D^{\dagger}$
}

\begin{abstract}
Background: For patients with nonobstructive azoospermia, sperm retrieval rates remain modest. We describe the use of optical coherence tomography to improve retrieval rates and to decrease tissue destruction.

Methods: Four patients underwent diagnostic testicular biopsy and imaging with the Niris optical coherence tomography device. We performed a descriptive comparison between optical coherence tomographic images and conventional histology.

Results: The measured seminiferous tubule diameter differed by $16 \mu \mathrm{m}$ between comparative imaging from optical coherence tomography and conventional histology using hematoxylin and eosin staining.

Conclusion: We illustrate the usefulness of optical coherence tomography in the setting of testicular biopsy and the management of nonobstructive azoospermia.
\end{abstract}

Can Urol Assoc J 2009;3(4):319-22

\section{Résumé}

Contexte : Chez les patients atteints d'azoospermie non obstructive, les taux de collecte de spermatozoïdes demeurent modestes. Nous décrivons le recours à une tomographie optique cohérente pour améliorer les taux de collecte et réduire la destruction tissulaire.

Méthodes : Quatre patients ont subi une biopsie testiculaire diagnostique et une épreuve d'imagerie à I'aide d'un appareil Niris de tomographie optique cohérente. Une comparaison descriptive a été effectuée entre les images obtenues par tomographie optique cohérente et les résultats des épreuves histologiques standard.

Résultats : La différence dans le diamètre des tubules séminifères mesuré par tomographie optique cohérente et par coloration histologique à l'hématoxyline-éosine n'était que de $16 \mu \mathrm{m}$.

Conclusion : Nous présentons une étude descriptive illustrant I'utilité de la tomographie optique cohérente pendant une biopsie testiculaire en vue de la prise en charge d'une azoospermie non obstructive.

\section{Introduction}

Optical coherence tomography (OCT) is an imaging modality that uses near-infrared light to create images analogous to B-mode ultrasonography; however, it meas- ures reflected infrared light from tissue structures rather than acoustic back-scattering. A laser is used to generate light, half of which is directed at the tissue and the other half at a mirror. The position of the mirror is altered in small increments until the reflections are "in phase." The amount of mirror movement combined with the amplitude of light produces a 2-dimensional image. ${ }^{1}$ Initially developed in 1991 at the Massachusetts Institute of Technology, OCT was first clinically applied to the eye where images were found to approach the resolution of histopathological specimens $(3-5 \mu \mathrm{m}){ }^{2}$ Although histology is viewed with a resolution of $3 \mu \mathrm{m}$, OCT images provide spatial resolution that is as high as 10-20 $\mu \mathrm{m}$. Within urology, OCT has shown promise in diagnosing bladder cancer and may have applications in the treatment of male infertility. ${ }^{3-5}$

For men with nonobstructive azoospermia (NOA), testicular sperm extraction (TESE) is most frequently used to retrieve sperm. Unfortunately, sperm are not found in $50 \%$ of these men. ${ }^{6}$ As a result, the concept of imagingassisted TESE has been investigated to improve retrieval rates while minimizing testicular trauma. The use of an operating microscope paved the way to improved success with the Microdissection TESE ${ }^{6}$ and recent studies with power-doppler ultrasound-guided TESE are encouraging. ${ }^{7}$ With its high resolution images, OCT may improve sperm retrieval rates by better identifying isolated foci of spermatogenesis in men with NOA. We sought to explore the applicability of OCT to TESE by first determining whether OCT images correlate well with parallel histology.

\section{Methods}

Four men presented to a male infertility clinic with suspected obstructive azoospermia. After we obtained Institutional Review Board approval, all patients underwent a diagnostic testicular biopsy between January and May 2006. All patients had an OCT examination (in vivo and ex vivo).

We performed OCT imaging using the US Food and Drug Administration (FDA)-approved Niris Imaging System (Imalux). Niris technology is based on common-path all-fibre interferometer topology. Near infrared light at 
$1310 \mathrm{~nm}$ is produced using a super luminescent diode. The light is directed to the tissue and the backscattered light is collected to produce a high spatial resolution image. Niris acquires real-time images with $200 \times$ 200 pixels, $15 \mu \mathrm{m}$ free space depth resolution $(11 \mu \mathrm{m}$ in tissue) and $25 \mu \mathrm{m}$ lateral resolution. We used a universal reusable and sterilizable $8 \mathrm{Fr}$ probe $(2.7 \mathrm{~mm}$ outer diameter) that allowed for 1.6-2.4 mm lateral scanning. We completed a single scan in 1.5 seconds. An internal measurement tool allowed us to examine real-time measurements of the tissue. In addition, the imaging software that we used with Niris (Presto 2005) allowed for measurements on saved images. The total cost of such a system is about US\$65 000. Optical coherence tomography technology is FDA class 1 equipment and has been used in previous studies with no adverse events noted. ${ }^{3-5}$

We made a transverse scrotal incision down through the tunica vaginalis to expose the surface of the testicle. We obtained initial images by placing the OCT probe directly on the surface of the tunica albuginea and directly on the seminiferous tubules to obtain multiple in vivo images. We performed the ex vivo OCT examination by placing the biopsy specimen on the tip of the probe and taking multiple images. We obtained cross-sectional images of the vas deferens in patients also undergoing vasovasostomy.

We then fixed the biopsy specimens in Hollande solution and embedded them in paraffin for sectioning. We used hematoxylin and eosin and trichrome stains routinely. A single pathologist performed microscopic evaluation using a magnification of 40-100. We visually compared OCT images with the histologic slides. We measured and compared the diameter of representative tubules from a cross-section of specimens, as viewed on histology and OCT.

\section{Results}

The average age of the 4 patients was 37 (31-44) years. Histology for the 4 patients revealed normal spermatogenesis. We obtained a total of 77 OCT images for all patients. The tunica albuginea could be distinctly seen when we placed the probe on its surface. Imaging below the tunica albuginea was less well defined given the high optical scattering property of the dense tunic (Fig. 1). We visualized tubules with improved resolution by directly imaging seminiferous tubules and incised tunica albuginea in all specimens (Fig. 2 and Fig. 3). We noted no thermal injury to the seminiferous tubules on gross visualization or on histology. The diameter of the average tubule was $218 \mu \mathrm{m}$ for OCT and and $212 \mu \mathrm{m}$ for histology among patients with normal spermatogenesis. The measured cross-sectional diameter seen on histology and OCT for all patients differed by an average of $16 \mu \mathrm{m}$. The vas deferens OCT images correlate very closely with histology findings (Fig. 4).

\section{Discussion}

Our descriptive study demonstrates that OCT can generate intraoperative images of scrotal structures such as seminiferous tubules, the epididymis and the vas deferens with a level of resolution that approaches that of histopathology (15 v. 3 microns). The technology is housed in a portable unit, the probe can be sterilized allowing for intraoperative use and images are acquired in real time.

Although OCT images can offer a resolution that is 100 times greater than that of ultrasound, the modality has several limitations. First, the depth of penetration is $2 \mathrm{~mm}$, which limits its ability to image deeply within tissues. This also restricts its use for transcutaneous imaging, which is commonplace with ultrasound. Second, the probe performs horizontal scans that take 1 second to process and display on the computer screen. This delayed image acquisition time creates motion artifact if the probe does not remain still within this 1 -second window. Modifications to the modality are reducing the image acquisition time.

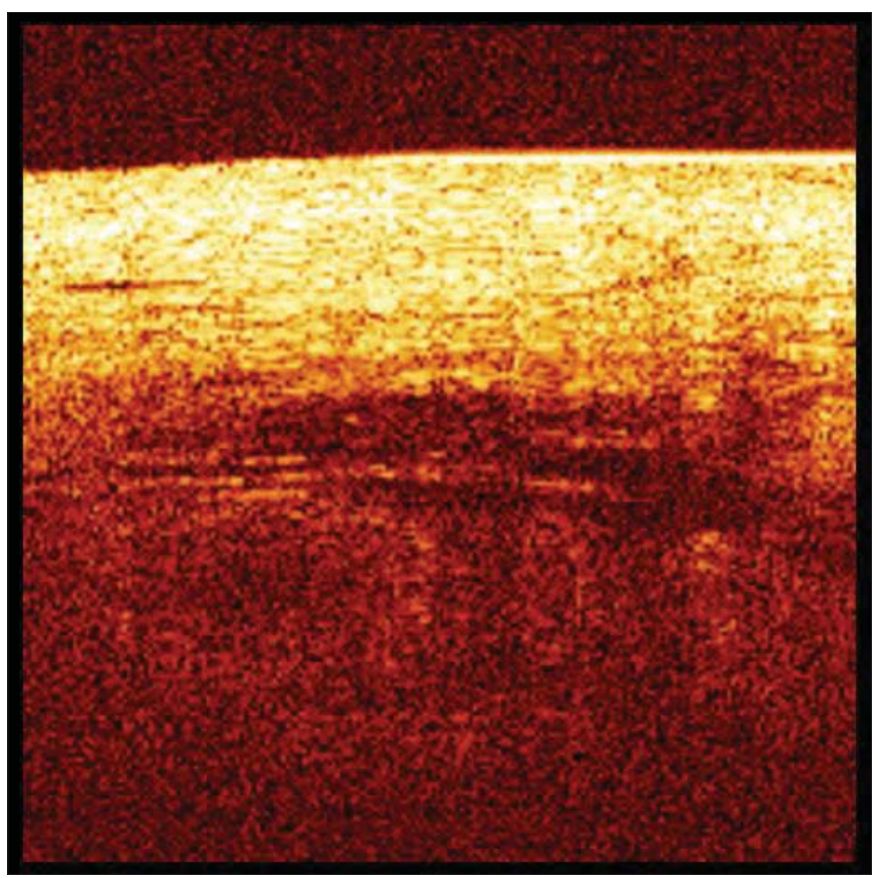

Fig. 1. Optical coherence tomography image of the testicle with the tunica albuginea intact. 

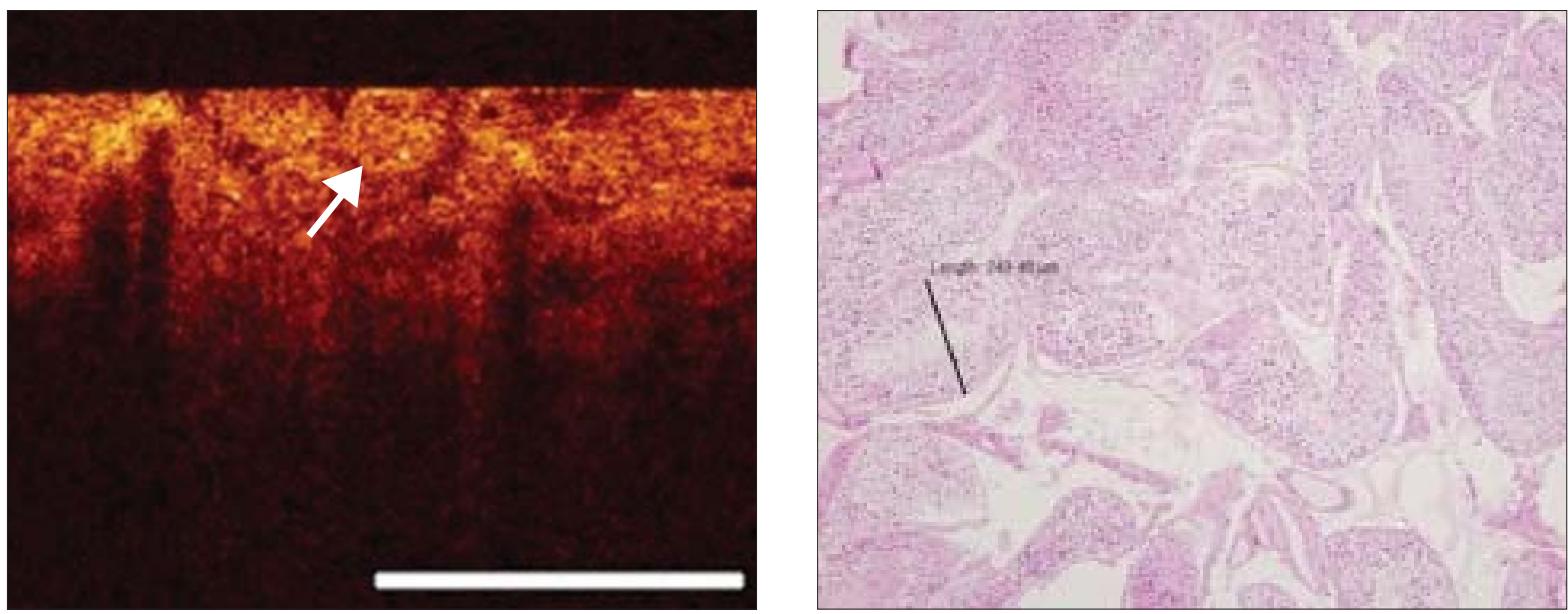

Fig. 2. Seminiferous tubules. The marked tubule diameter is $250 \mu \mathrm{m}$ (1-mm reference marker on the optical coherence tomography image).
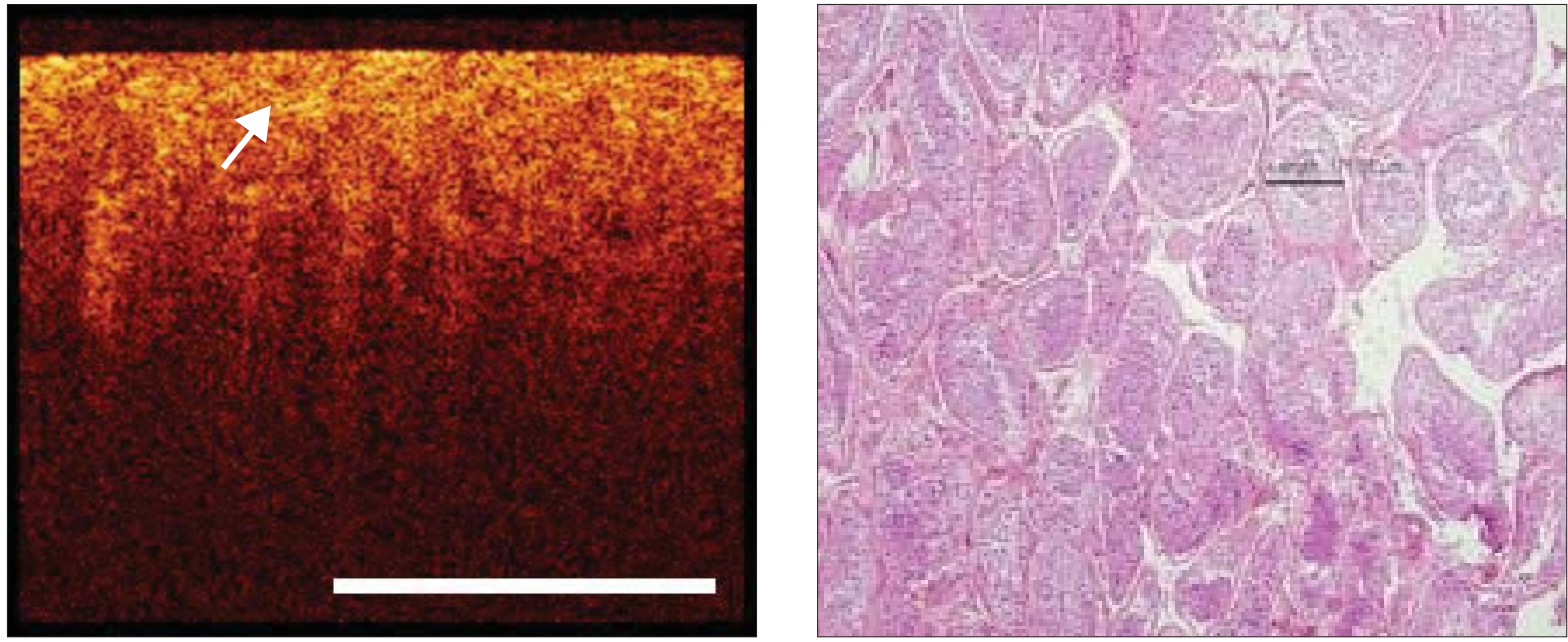

Fig. 3. Seminiferous tubules. Arrows indicate tubules. The marked tubule size is $172 \mu \mathrm{m}$ (1-mm reference marker on the optical coherence tomography image)
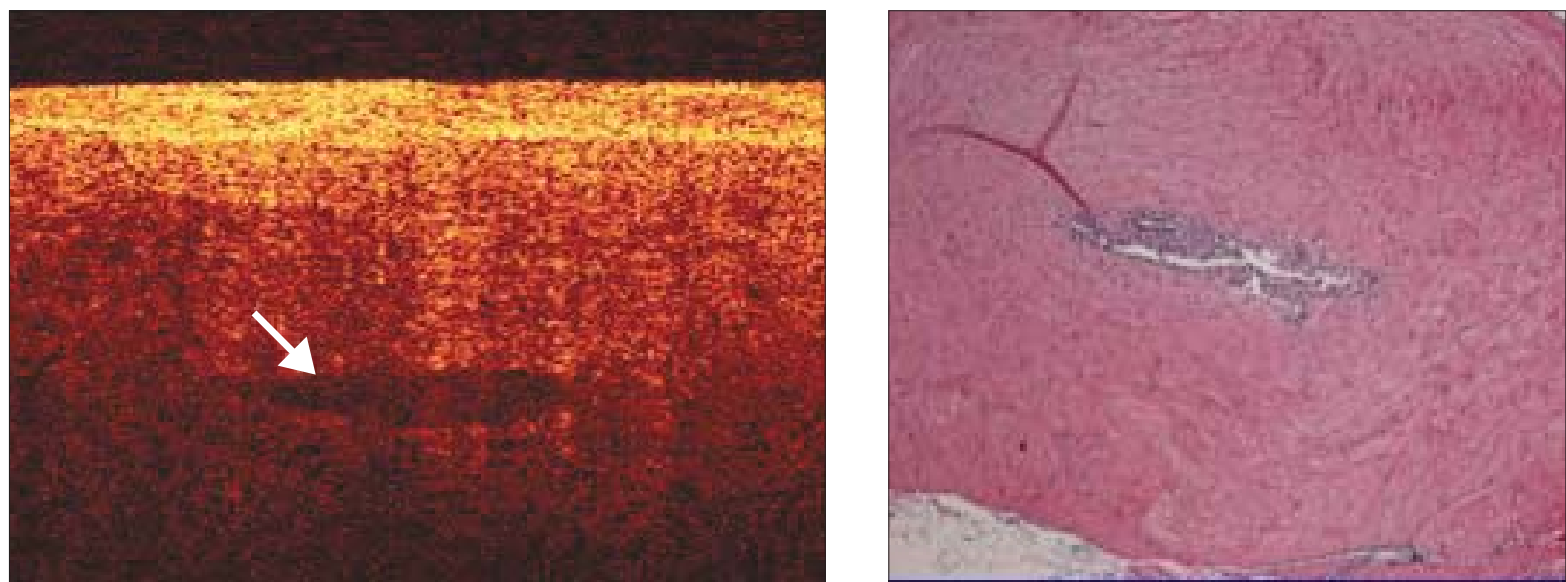

Fig. 4. Vas deferens. Arrow indicates the lumen. 
Finally, experience is required to overcome the short learning curve for interpreting OCT images, which in our case proved to be about 30 scanning sequences for testicular tissue and fewer for more prominent structures such as the vas deferens and the epididymis.

The OCT images of the vas deferens were able to delineate all layers of the vas deferens. Intraluminal cautery used during a vasectomy may create areas where the lumen is scarred while the outer layers are viable. As a result, knowing when the vas deferens has been cut back to a level where all layers are viable can be a challenge. Using OCT may help optimize anastamotic patency with vasectomy reversal by ensuring a viable vas deferens throughout its thickness. Epididymal tubules can also be individually imaged, which may be a useful surgical adjunct during a vasoepididymostomy. By surface scanning through the thin epididymal tunic, the transition point correlating with the level of epididymal obstruction can be better identified to allow the anastomosis to be performed at the most distal epididymal level possible. Future studies addressing whether this would provide added value for male fertility microsurgeons are necessary and would translate to improved patency rates.

The goal of TESE for men with NOA continues to be optimal sperm retrieval rates with minimal testicular trauma. Although microdissection TESE (Micro-TESE) has yielded the highest retrieval rates, it still requires 1 or more tunical incisions and a thorough microsurgical exploration of the testicle. ${ }^{6}$ Although OCT can image individual seminiferous tubules, several obstacles must be overcome before it can successfully be used as a surgical adjunct for TESE. First, the high reflective index of the tunical albuginea further limits the depth to which OCT images can be acquired. As a result, a tunical incision is still required to visualize all seminiferous tubules, which affords little advantage over a standard Micro-TESE. Sec- ond, although OCT could be used to measure the diameter of an individual tubule to identify the larger tubules that may have sperm, a simple intraoperative micrometer has been shown to provide the same benefits. Finally, more studies will be necessary to determine whether OCT can differentiate various histologies such as maturation arrest, hypospermatogenesis, Sertoli-cell and normal spermatogenesis. This would be an important stepping stone toward the ideal scenario of being able to image and target specific tubules containing sperm with minimal testicular trauma.

* Urologic Oncology Fellow, University of California, San Francisco, Calif., and †Assistant Professor, University of New Mexico, One University of New Mexico, Albuquerque, NM

This article has been peer reviewed.

Competing interests: None declared.

\section{References}

1. Crow P, Stone N, Kendall C, et al. Optical diagnostics in urology: current applications and future prospects. BJU Int 2003;92:400-7.

2. Huang D, Swanson E, Lin C, et al. Optical coherence tomography. Science 1991;254:1178-81.

3. Tearney GJ, Brezinski M, Southern J, et al. Optical biopsy in human urologic tissue using optical coherence tomography. J Urol 1997;157:1915-9.

4. Zagaynova E, Streltsova 0, Gladkova N, et al. In vivo optical coherence tomography: feasibility for bladder disease. J Urol 2002;167:1492-6.

5. Manyak MJ, Gladkova N, Makari J, et al. Evaluation of superficial bladder transitional cell carcinoma by optical coherence tomography. J Endourol 2005;19:570-4.

6. Schlegel PN. Testicular sperm extraction: microdissection improves sperm yield with minimal tissue excision. Hum Reprod 1999;14:131-5.

7. Har-Toov J, Eytan 0, Hauser R, et al. A new power Doppler ultrasound guiding technique for improved testicular sperm extraction. Fertil Steril 2004;81:430-4.

Correspondence: Dr. Cole Davis, 1600 Divisadero St., 6th floor, Box 1695, San Francisco CA 94143; daviscb@urology.ucsf.edu 